\title{
Hans Haacke, Andrea Fraser und das Projekt einer gegenhegemonialen Disziplingeschichte
}

\author{
Markus Rieger-Ladich
}

Online publiziert: 25. Juni 2020

(C) Der/die Autor(en) 2020

Zusammenfassung Hans Haacke und Andrea Fraser, zwei zeitgenössische Künstler*innen, haben mit ihren Arbeiten die Selbstkritik des künstlerischen Feldes immer weiter radikalisiert - und sie werfen dabei Fragen auf, die auch für Vertreter*innen der (Erziehungs-)Wissenschaft von besonderer Brisanz sind. Etwa danach, wie sich ein patriarchaler Apparat kritisieren lässt, dessen Teil man*frau ist. Vor diesem Hintergrund stelle ich das Projekt einer gegenhegemonialen Disziplingeschichte vor, skizziere seine Konturen und kontextualisiere es. Und versuche dabei zugleich zu zeigen, dass die künstlerische Institutionskritik die wissenschaftliche Selbstbefragung zu stimulieren vermag. Der Blick über die Grenzen der Disziplin ist - auch in diesem Fall - überaus lohnenswert.

Schlüsselwörter Künstlerkritik · Institutionskritik · Disziplingeschichte · Gegenhegemonie 


\title{
Hans Haacke, Andrea Fraser and the project of a counter-hegemonic history of discipline
}

\begin{abstract}
With their works, Hans Haacke and Andrea Fraser, two contemporary artists, have increasingly radicalized the self-criticism of the artistic field-and in doing so they raise questions that are also of particular relevance to representatives of (educational) science. For example, how to criticize a patriarchal apparatus of which one is a part. Against this background, I present the project of a counterhegemonic history of discipline, sketching its contours and contextualizing it. At the same time, I try to show that artistic institutional critique is capable of stimulating scientific self-interrogation. A look beyond the boundaries of the discipline is-also in this case-extremely worthwhile.
\end{abstract}

Keywords Artistic institutional critique $\cdot$ History of discipline $\cdot$ Counter-hegemony

Ein Heft, das sich ausdrücklich der Transdisziplinarität verschreibt, ist eine willkommene Gelegenheit, eine neue Form wissenschaftlicher Reflexivität zu erproben - und zu diesem Zweck den Blick auf zwei zeitgenössische Künstler*innen zu werfen. Obwohl sich die Felder der Wissenschaft und der Kunst unterschiedlichen sozialen Praktiken verdanken, obwohl sie verschiedene Semantiken pflegen und ihr Personal auf differente Weise ausbilden, ist ihnen doch manches gemeinsam: Wissenschaftler*innen wie Künstler*innen leisten unverzichtbare Beiträge zur Selbstthematisierung der Gesellschaft. Sie sensibilisieren für die Macht von Narrativen und die Effekte von Diskursen; sie durchkreuzen dominante Erzählungen, indem sie einflussreiche gesellschaftliche Akteur*innen beim Beobachten beobachten. Die Akteur*innen aus Wissenschaft und Kunst reichern somit nicht allein die zirkulierenden Beschreibungsversuche mit Komplexität an; sie steigern auch deren Reflexivität. Und sie verweisen damit auf die Kontingenz des Sozialen (vgl. Baecker 2017; Rustemeyer 2017).

Wissenschaft und Kunst erinnern mithin daran, dass die Bedingungen unter denen wir leben nicht schicksalhaft sind. Sie konfrontieren uns damit, dass wir in menschengemachten Verhältnissen leben (vgl. Dath 2018). Mit ihren Beiträgen - gleich, ob es sich um empirische Studien handelt, um theoretische Abhandlungen oder um künstlerische Arbeiten - kündigen sie gewissermaßen die Orientierung am Status quo auf. Sie demonstrieren, einmal im Modus des Arguments, ein anderes Mal im Modus des Zeigens (vgl. Gabriel 2019; Rieger-Ladich 2014), dass soziale Arrangements und kulturelle Ordnungen nicht unangreifbar sind; sie zeigen, dass wir diese auch anders organisieren können. Wissenschaft und Kunst schulen dergestalt den „Möglichkeitssinn“ (Musil) und ein Denken in Alternativen. Anders formuliert: Sie erweisen, dass das, was wir als natürlich und schicksalhaft erleben, häufig genug die Folge von Entscheidungen ist; sie erweisen vermeintlich natürliche Ordnungen als bloß gestiftete (vgl. Barthes 2012).

Eines meiner gegenwärtigen Forschungsinteressen besteht darin, neue Formen der Disziplingeschichtsschreibung zu erproben. Auch aus diesem Grund interessiere ich mich für Projekte wissenschaftlicher und künstlerischer Art, die sich um eine 
gesteigerte Form der Reflexivität bemühen. Mein Augenmerk gilt daher solchen Unternehmungen, welche die eigene Praxis beobachten, die ihre Energien also weniger auf Praktiken der Legitimierung verwenden, sondern die Selbstbefragung forcieren. Nachdem ich mich in der Vergangenheit bereits Vertreter*innen der Soziologie, der Cultural Studies und der Postcolonial Studies zugewandt habe (vgl. Rieger-Ladich 2019), werde ich im Rahmen dieses Beitrags zwei zeitgenössische Künstler*innen vorstellen. Hans Haacke und Andrea Fraser sind prominente Vertreter*innen dessen, was innerhalb der Kunst „Institutionskritik“ genannt wird. Sie haben mit ihren Arbeiten die Selbstkritik des künstlerischen Feldes immer weiter radikalisiert - und dabei Fragen aufgeworfen, die auch für uns, als Vertreter*innen der (Erziehungs-)Wissenschaft, von Brisanz sind. Und das insbesondere für jene Fachvertreter*innen, die sich dafür interessieren, welche Rolle der Disziplingeschichtsschreibung bei der kritischen Selbstbefragung der erziehungswissenschaftlichen Disziplin zukommt bzw. künftig zukommen sollte. Gemeinsam mit meinen Tübinger Kolleginnen Anne Rohstock und Karin Amos habe ich unlängst den Vorschlag gemacht, Erinnern und Vergessen als machtvolle Praktiken der Disziplinpolitik zu betrachten (vgl. RiegerLadich et al. 2019). Dieses Projekt einer gegenhegemonialen Disziplingeschichte werde ich im zweiten Teil meiner Überlegungen vorstellen. Es ist Teil einer größeren Bewegung, die sich seit einigen Jahren schon dafür einsetzt, endlich eine Geschichtsschreibung zu überwinden, welche die Heroisierung weißer, westlicher und männlicher Wissenschaftler betreibt. Diese Unternehmen, komplexere Erklärungen zu entwickeln, lassen sich auch innerhalb der Philosophie, der Soziologie, der Literaturwissenschaft sowie der Wissenschaftstheorie beobachten. Sie gehen ungleich kontextsensibler, kleinteiliger und facettenreicher vor, als dies für die konventionelle Form der Disziplingeschichtsschreibung typisch ist (vgl. etwa Flasch 2008). Ihnen gilt das Streben nach Erkenntnis als eine anspruchsvolle soziale Praxis, die sich in komplexen Konstellationen vollzieht, die hybride Arrangements kennt sowie unterschiedlich machtvolle Akteur*innen (vgl. Haraway 1995; Fleck 1980).

Bevor ich mich in meinem Beitrag dem Feld der Kunst zuwende und mit Hans Haacke und Andrea Fraser zwei bedeutende Vertreter*innen der zeitgenössischen Institutionskritik vorstelle, sei in einer Vorbemerkung das Anliegen dieser Kunstrichtung skizziert. Nach den knappen Porträts wende ich mich dem Feld der Wissenschaft zu und suche zu erläutern, was die wissenschaftliche Selbstreflexion von der künstlerischen Institutionskritik lernen kann. Die These, die ich dadurch zu plausibilisieren suche, lautet: Die künstlerische Institutionskritik kann die wissenschaftliche Selbstbefragung stimulieren und deren Intensivierung befördern. Ich halte das für dringend geboten und es daher lohnenswert, sich auch als Vertreter*in der Erziehungswissenschaft mit den Arbeiten von Haacke und Fraser näher zu befassen.

Doch zunächst ein Hinweis auf die Institutionskritik: Sie geht hervor aus einer Bewegung, in der Künstler*innen ganz gezielt die Bedingungen thematisieren, unter denen sie arbeiten. Sie wenden sich, einsetzend in den späten 1960er und frühen 1970er Jahren, in kritischer Absicht jenen Institutionen und Akteur*innen zu, welchen das künstlerische Feld seine Existenz verdankt (vgl. Brüggmann 2020; Bourdieu 1999; Marchart 2005). Die künstlerische Praxis wird von ihnen daher gleichsam im Modus der Reflexivität betrieben: Zum Gegenstand werden die Logiken des künstlerischen Feldes selbst (vgl. Raunig 2007; Steyerl 2006). Auch wenn 
diese Form einer mitlaufenden Selbstbeobachtung der modernen Kunst durchaus nicht unbekannt ist, war doch die Radikalität, mit der dies nun betrieben wurde, neu. Und schon bald führte sie zu einem ersten Skandal. Dieser kann als die Geburtsstunde, als die Initialzündung der Institutionskritik gelten (vgl. van den Berg 2015).

\section{New York City, 1971}

1971 wird vom renommierten Guggenheim-Museum eine Ausstellung von Hans Haacke angekündigt. Hans Haacke, in Deutschland aufgewachsen und seit Mitte der 1960er Jahre in New York lebend, hat hierfür eine größere Zahl von Fotografien ausgewählt. Diesen sind kurze Texte zugeordnet, die neben ihnen angebracht werden sollen. Insgesamt sind 146 Fotografien vorgesehen, auf denen New Yorker Gebäude abgebildet sind. Doch einen Monat vor dem geplanten Start wird sie abgesagt. Und bald darauf der verantwortliche Kurator der Ausstellung, Edward F. Fry, entlassen, weil dieser sich für Haacke eingesetzt hatte.

Was war geschehen? Was hatte es mit den Gebäuden auf sich, die auf den Fotografien zu sehen waren? Und was mit den Texttafeln, die neben ihnen zu sehen sein sollten? Und zu welchem Zweck sollten Stadtpläne von Harlem und der Lower East Side aufgehängt werden?

Haacke hatte sich für die Verslumung Manhattans zu interessieren begonnen und für die Transaktionen eines Immobilienkonsortiums. Er hatte Informationen aus Datenbanken zusammengetragen - und fein säuberlich dokumentiert, wer zu welchem Preis welche Immobilie erstanden hatte. Und diese nun verfallen lies. Das zeigten die Fotos und das erläuterten die Texttafeln. Und um sich von der Dimension des Skandals eine Vorstellung machen zu können, hatte Haacke zwei Stadtpläne vorbereitet. Der Titel der geplanten Ausstellung lautete: „Shapolsky et al. Manhattan Real Estate Holdings, a Real-Time Social System, as of May 1, 1971“. Selbst als Haacke zusicherte, die Angaben zu anonymisieren, so dass kein Rechtsstreit zu befürchten war, willigte der Direktor des Guggenheim Museums nicht ein. Er, H. Haacke, habe die Grenzen der Kunst überschritten; überdies schließe die Satzung des Museums „soziale und politische Ziele“ aus (zit. nach van den Berg 2015, S. 172). Schon bald kam es zu Protestaktionen anderer Künstler*innen. H. Haacke machte aus der Korrespondenz mit dem Direktor ein Buch - „Shapolksy et al.“ wurde in anderen Museen gezeigt und schließlich zu einer der meistdiskutierten Arbeiten der frühen 1970er Jahre.

Diese Arbeit H. Haackes kann als exemplarisch gelten für die erste Phase der institutional critique. H. Haacke wendet sich mit dem Museum der wichtigsten Institution im künstlerischen Feld zu (vgl. Bourdieu und Haacke 1995). Er macht dabei auch vor Geldgebern und Board-Mitgliedern nicht halt, interessiert sich für deren Vermögensverhältnisse und problematisiert den Einfluss der Ökonomie auf das Kunstfeld. Das Pathos der Distanz, das in H. Haackes Arbeit zum Ausdruck kommt, ist typisch für die erste Generation der Institutionskritik. Die Künstler*innen erscheinen hier als das Gegenüber zum Kunstfeld, das mehr und mehr von den Gesetzen des Marktes regiert wird. Im Zentrum stehen daher - in den 1970er Jahren 
- die Museen und Galerien. Hier kommt es zur Akkumulation von Kapital, hier trifft die Finanzaristokratie auf die Macht der Politik - und alle sind vom Glamour des Kunstfeldes angezogen. Museen und Galerien stehen daher für die Verunreinigung des Kunstfeldes. Sie sind das Einfallstor für fremde Interessen und die Einflussnahme von außen (vgl. von Hantelmann und Meister 2010).

Das Abrücken von dieser Geste der Distanznahme kennzeichnet die zweite Phase der Institutionskritik. Sie setzt Ende der 1980er Jahre ein, als jüngere Künstler*innen sich mit Arbeiten von Hans Haacke, Daniel Buren und anderen Vertretern der ersten Phase auseinandersetzen - dabei aber nun andere Akzente setzen (vgl. Brüggmann 2020, S. 75-96). Sie interessieren sich zwar immer noch für die Einrichtungen des Kunstfeldes, greifen dabei aber ungleich seltener auf dichotome Figuren zurück. Sie machen die eigene Situiertheit zum Thema und gehen den unterschiedlichen Formen ihrer Involviertheit in das Kunstfeld nach. Sie bemühen also kein Gegenüber, dessen Versuche der Einflussnahme es abzuwehren gilt, sondern nehmen sich selbst als Akteur*innen des Kunstfeldes in den Blick. Der Kunsttheoretiker Gerald Raunig (2007) hat das bündig resümiert: ,Schematisch formuliert, ging es der ,ersten Generation ' der Institutionskritik um Distanz von der Institution, der ,zweiten “ um die unausweichliche Involviertheit in die Institution“" (Raunig 2007, S. 88).

\section{Philadelphia, 1989}

Andrea Fraser, geboren 1965 und ausgebildet unter anderem an der New York University, ist beides: Künstlerin und Intellektuelle, Aktivistin und Kunsttheoretikerin. Sie muss deshalb zu den exponierten Vertreter*innen dieser zweiten Generation der Institutionskritik gezählt werden, weil sie im Aufdecken dieser Involviertheit des Kunstfeldes so unnachgiebig und schonungslos ist, wie kaum eine andere. Bekannt wurde sie mit Performances, die darauf abzielen, jene sozialen Praktiken, denen Museen und Galerien ihre Macht verdanken, aufzudecken. Sie bringt die Rituale des Kunstfeldes zur Wiederaufführung - und sucht ihnen auf diese Weise ihre performative Kraft zu nehmen.

So etwa in „Museum Highlights: A Gallery Talk“ aus dem Jahr 1989 (vgl. van den Berg 2015). In ein tailliertes Kostüm gekleidet, die Haare zusammengebunden und mit einer Hornbrille ausgestattet, führt sie durch das Philadelphia Museum of Art. Sie gibt sich als Dozentin mit dem Namen Jane Castleton aus und stattet während ihrer Führung unterschiedlichen Räumen und Einrichtungsgegenständen des Museums einen Besuch ab - dem Umkleideraum etwa und den Toiletten. Sie würdigt einen Wasserspender und bleibt vor einem Exit-Schild stehen, um zu erklären, dass dieses die Handschrift eines brillanten Künstlers erkennen lasse. Fraser lenkt das Interesse auch auf Feuerlöscher und Luftbefeuchter - und stört damit die andächtige Stimmung, die in Ausstellungsräumen zumeist herrscht (vgl. Bennett 2008). Sie irritiert das Publikum, persifliert die Führung und zeigt das Museum als autoritäre Einrichtung. A. Fraser wiederholt also in ihrem „Gallery Talk“ die Praktiken der Autorisierung in subversiver Absicht. Sie streut Sand ins Getriebe der gut geölten Distinktionsmaschine. 
Kaum weniger wichtig als ihre Performances sind die Texte, in denen A. Fraser ihre künstlerische Praxis reflektiert. Dabei wird deutlich, dass ihr H. Haackes Neigung zu einer topologischen Metaphorik, die mit dem Dual von Innen und Außen arbeitet, fremd ist. Bei aller Wertschätzung für dessen Arbeiten, verzichtet sie weitgehend auf solche Oppositionsbildungen: „Just as art cannot exist outside the field of art, we cannot exist outside the field of art, at least not as artists, critics, curators, etc. [...] So if there is no outside for us, it is not because the institution is perfectly closed, or exists as an apparatus in a ,totaly administered society ' [...]. It is because the institution is inside of us, and we can't geht outside of ourselves“" (Fraser 2005a, S. 282).

A. Fraser radikalisiert somit die künstlerische Selbstbefragung - und greift zu diesem Zweck auch auf Arbeiten des französischen Soziologen Pierre Bourdieu zurück. Intensiv studiert sie zunächst die „Feinen Unterschiede“ (Bourdieu 1987) und später dessen Studien zum Kunstfeld (vgl. Bourdieu 1999). Es ist nicht zuletzt P. Bourdieus Theorie sozialer Felder, welche A. Fraser, die sich zunächst mit feministischen und psychoanalytischen Theorien befasst hatte, dazu bringt, die Einrichtungen des kulturellen Feldes auf eine neue Weise in den Blick zu nehmen: „Bourdieus Theorie verschiebt die Frage danach, außerhalb irgendeiner totalisierten ,Kunstinstitution * oder gegen eine solche zu sein, dezidiert zu viel spezifischeren, historischen und materiellen Fragestellungen nach der Art der Institution, nach der Art der Werte, die sie strukturieren, und nach den Formen von Macht, die sie reproduziert" (Fraser 2008, S. 296).

A. Fraser löst sich also davon, auf eine abstrakte Weise für die „Autonomie der Kunst“ zu werben oder diese einzufordern; sie entscheidet sich stattdessen für kleinteiligere Analysen. Sie untersucht konkrete Einrichtungen, studiert die Machtverhältnisse vor Ort. Sie schlüpft also nicht in die Rolle der unbeteiligten Beobachterin oder der scharfsinnigen Kritikerin, sondern sucht die Komplizenschaft zwischen Kunst und Ökonomie sichtbar zu machen. Und muss dabei feststellen, als sie eine Arbeit für die Generali Foundation in Wien vorbereitet, dass die Geldgeber längst begriffen haben, dass sie sich erst dann mit einem Kunstprogramm schmücken können, wenn sie den Künstler*innen völlige Freiheit zugestehen. Die „Autonomie“, so Fraser, ,war geradezu die Bedingung der Ausbeutung der Kunst zum Zweck der Öffentlichkeitsarbeit, die Grundlage der Legitimierung, welche der Konzern sich dann als symbolischen Profit aneignen konnte“ (Fraser 2008, S. 299).

Spätestens an diesem Punkt, Mitte der 1990er Jahre, wird deutlich, dass die institutional critique in die Krise geraten ist und vor zwei großen Herausforderungen steht. Sie droht, erstens, zu einem Opfer ihres eigenen Erfolges zu werden. Institutionskritik, die doch angetreten war, den Apparat des Kunstfeldes zu stören und dessen Abläufe zu unterbrechen, hat sich zu einem eigenständigen Genre entwickelt. Sie ist längst eine eigene Marke. Und als solche ist sie, zweitens, eine attraktive Kandidatin für die PR-Abteilungen großer Konzerne. „Künstlerische Freiheit“, so Fraser, ,erwies sich als nichts anderes als eine weitere Form von Macht und Privileg“ (Fraser 2008, S. 299). Sie verkauft sich meistbietend, sie prostituiert sich - und Andrea Fraser wäre nicht die begnadete Performerin, die sie ist, wenn sie nicht auch das längst zum Gegenstand einer Performance gemacht hätte. Das Video „Untitled“ aus dem Jahr 2003 zeigt sie beim Sex mit einem anonymen Kunstsammler, der dafür 
20.000 Dollar bezahlte und im Gegenzug die Videoarbeit erwarb (vgl. van den Berg 2015, S. 174).

Seither wird intensiv diskutiert, wie eine radikale ästhetische Praxis aussehen kann. Lässt sich noch in einer anspruchsvollen Weise von „Opposition“ und von „Intervention“ sprechen, wenn sich dieser radical chic gut verkauft und wenn sich Versicherungskonzerne einen Kunstraum gönnen, in dem sie diese Arbeiten ausstellen? In bester Innenstadtlage und edlem Ambiente - wie die Wiener Generali Foundation? Wie lässt sich von „Kritik“ sprechen, wenn sie zu einem Label zu werden droht und auf raffinierte Weise vereinnahmt wird (vgl. Draxler 2007; Boltanski und Chiapello 2003)?

\section{Zwischenbemerkung}

Die vielleicht wichtigste Reaktion auf diese Entwicklung besteht in der Selbstkritik der Institutionskritik. Was als eine Rückwendung auf die Arbeit innerhalb des Kunstfeldes begonnen hatte, in den 1970ern, steht nun vor der Herausforderung, die Reflexivität noch weiter zu steigern. Und genau das geschieht gegenwärtig. Derzeit diskutieren Künstler*innen und Kunsttheoretiker*innen wie Andrea Fraser und Hito Steyerl, Isabell Graw, Gerald Raunig und viele andere, was es bedeuten kann, noch länger von Kritik im Kunstfeld zu sprechen. Sie weisen darauf hin, dass es einem paradoxen Unternehmen gleicht, wenn die Kritik selbst zu einer Institution wird. Anders formuliert: Wie lässt sich Institutionskritik unter den Bedingungen des 21. Jahrhundert buchstabieren? Wie lassen sich „Gesellschaftskritik, Institutionskritik und Selbstkritik“ miteinander verknüpfen (Raunig 2007, S. 91)? Und in ein Spannungsverhältnis zueinander versetzen, so dass sie sich wechselseitig stimulieren?

Es sind Fragen dieser Art, die auch für das Wissenschaftsfeld von Interesse sind - insbesondere dann, wenn wir uns für Praktiken des Archivierens und des Speicherns interessieren und Fragen nach dem Gedächtnis einer Disziplin aufwerfen. Dabei kann als unstrittig gelten, dass auch im Feld der Wissenschaft die Frage der Kritik seit jeher überaus prominent ist (vgl. Sonderegger 2019; Reitz 2016). Auch innerhalb des wissenschaftlichen Feldes gibt es einflussreiche Gate-Keeper und Instanzen der Konsekration, gibt es Zentralorgane und einen Kanon, der die sogenannten einschlägigen Texte verzeichnet. Aber auch hier, im Feld der Wissenschaft, wächst in der jüngsten Zeit die Kritik an den Praktiken der Beglaubigung und das Interesse an Erzählformen, die den Kult um die unerreichten Genies und die einsamen Meisterdenker hinter sich lassen. Die Logik des Feldes wird daher auch innerhalb der Wissenschaft befragt. Auch hier, in der Academia, lässt sich mithin eine forcierte Selbstbefragung beobachten, wird um Reflexivität gerungen (vgl. Rieger-Ladich 2009).

Lange Zeit wurden diese Impulse abgewehrt. Aber nun, so scheint es, tut sich etwas. Die Selbstbefragung in kritischer Absicht nimmt Fahrt auf und die Stimmen derer, die - psychoanalytisch gesprochen - das ,akademische Unbewusste“ (Bourdieu 1984) zum Gegenstand machen wollen, mehren sich. Dafür spricht etwa die Einrichtung einer Kommission „Wissenschaftsforschung“ im Jahr 1985 durch die 
Deutsche Gesellschaft für Erziehungswissenschaft (DGfE). Fraglos ist diese Kommission nicht der einzige Ort, an dem es zu einer systematisch angeleiteten Selbstbeobachtung kommt, aber sie hat den Vorteil, dass sie institutionalisiert ist - und daher nicht von den Befindlichkeiten und Zeitressourcen einzelner abhängt. Das Streben nach Reflexivität hat also längst einen Ort, auch innerhalb unserer Fachgesellschaft.

Wir stehen demnach - im Kunst- wie auch im Wissenschaftsfeld - vor derselben Herausforderung: Wie lässt sich ein patriarchaler Apparat kritisieren, dessen Teil man ist? Dem die Akteur*innen - auf einer symbolischen, aber auch materiellen Ebene - ihre Existenz verdanken. Und einen großen Teil ihrer Privilegien. Anders formuliert: Wie lässt sich eine hegemoniale Ordnung destabilisieren, deren Element man ist? Wie im Namen einer Autorität sprechen, die man*frau zu untergraben sucht? Wie müsste eine Praxis der Kritik aussehen, die weitgehend frei ist von einer selbstgerechten Attitüde, die um die eigene Involviertheit weiß - und die sich nicht vollständig zum Komplizen des Status quo macht (vgl. Rieger-Ladich 2020)? Diesen Fragen wende ich mich im zweiten Teil meines Beitrags zu.

\section{Paris, 1984}

Wie eng die Verbindung zwischen Wissenschaft und Kunst bisweilen ist, wurde bei einem Vortrag deutlich, der 1984 in Paris stattfand. Fünf Jahre vor Andrea Frasers „Gallery Talk“ kam es im Quartier Latin zu einer Performance, die für viele der Anwesenden ähnlich irritierend war wie jene in Philadelphia. Das Collège de France, eine der renommiertesten Bildungseinrichtungen Frankreichs, hatte zur Antrittsvorlesung von Pierre Bourdieu eingeladen. Worüber der Soziologe sprechen würde, blieb etwas unklar. Der Titel der Antrittsvorlesung lautete: „Leçon sur la leçon“ (Bourdieu 1995). Und doch war genau das Bourdieus Thema. Er sprach in seiner Antrittsvorlesung über das Format der Antrittsvorlesung. Er thematisierte also ein Ritual des wissenschaftlichen Feldes und entzauberte den weihevollen Moment der Aufnahme. Er nahm dem Akt der Inthronisierung seinen Glanz - und bediente genau damit das Format. Bourdieu gab, wie verlangt, einen Einblick in aktuelle Forschungsschwerpunkte und lies erkennen, welche Themen er in der Zukunft zu erforschen beabsichtige. Und dies war sein Programm: Die Arbeit an einer reflexiven Soziologie, die die Waffen der Kritik auch gegen sich selbst richtet.

Dieses Projekt wird freilich nicht allein von P. Bourdieu verfolgt. Der Poststrukturalismus und der Neopragmatismus, die Cultural Studies und die Postcolonial Studies verdanken sich ähnlichen Impulsen. Eine solche radikalisierte Form wissenschaftlicher Selbstkritik kann nicht umhin, auch über alte und neue Varianten der Disziplingeschichtsschreibung nachzudenken. Dies ist deshalb zwingend, weil die wissenschaftliche Praxis auf Begriffe und Wissensbestände, auf Methoden und Theorien zurückzugreifen gezwungen ist, die in der Vergangenheit entwickelt wurden und das Gedächtnis der Disziplin darstellen. Da dieses Gedächtnis stets selektiv verfährt, werden nie sämtliche Erkenntnisse vorgehalten und erinnert. Die Zukunft der Erziehungswissenschaft als einer kritischen Sozialwissenschaft hängt somit auch daran, auf welche Weise sie ihr Gedächtnis organisiert, ihre Archive anlegt und welche Vergangenheit(en) sie rekonstruiert. 
Welche Gestalt müsste die Disziplingeschichte der Erziehungswissenschaft annehmen, wenn sie im Dienst der hier skizzierten kritischen Selbstbefragung steht? Welche Form müsste das Speichern und Archivieren annehmen, wenn man die hegemonialen Kämpfe in Rechnung stellt, die um die Ordnung des pädagogischen Diskurses geführt werden? Wie lässt sich der fortwährende Widerstreit darstellen, der Dissens abbilden? Wie lassen sich Grenzziehungen rekonstruieren? Und wie sollte das disziplinäre Gedächtnis gepflegt werden, um künftig die Zahl der theoretischen Optionen zu erhöhen, die pädagogische Reflexion mit Komplexität anzureichern und die Entwicklung neuer, bislang unbekannter Denkstile und Reflexionsformen gezielt zu fördern?

Lange Zeit war die Disziplingeschichte ein Instrument der Identitätspolitik. Der Blick zurück auf die eigenen Anfänge geschah kaum einmal unmotiviert: In die Konkurrenz mit anderen Disziplinen verstrickt, suchten Fachvertreter*innen das Gewicht der eigenen Disziplin dadurch zu erhöhen, dass sie einen imposanten Stammbaum freilegten und renommierte Gründerväter identifizierten. Der Ausweis der ,Anciennität“ galt dabei als Quelle der Reputation; er diente, so Wolf Lepenies (1978), dem „Legitimationserwerb“ sowie der „Identitätsstärkung“ (Lepenies 1978, S. 449).

Seit etwa zwei Dekaden werden nun die Stimmen derer vernehmbar, die Disziplingeschichte nicht länger in diesem rückwärtsgewandeten Modus betreiben wollen (vgl. etwa Rieger-Ladich 2010; Thiel 1993; Rustemeyer 1992). Sie setzen sich dafür ein, Disziplingeschichte als eine Form der kritischen Selbstvergewisserung zu betreiben; ihnen gilt die rückhaltlose Selbstbefragung der Geschichte der fachspezifischen Kommunikation als Chance, eine reflexive Wissenschaftspraxis zu entwickeln. Besondere Impulse verdankt dieses Unternehmen zwei Erlanger Forschungsprojekten. Volker Peckhaus und Christian Thiel (1999) sprechen sich in diesem Zusammenhang nachdrücklich für einen Perspektivenwechsel aus:

Indem die kontextuelle Disziplingeschichtsschreibung den einzelnen Wissenschaftler in ein Geflecht der auf ihn wirkenden Einflüsse sowohl wissenschaftlicher als auch nichtwissenschaftlicher Art einbindet, z. B. in das Netz wissenschaftlicher, gesellschaftlicher und privater Kommunikation, kommen auch Persönlichkeiten des zweiten und dritten Gliedes als konstitutiv für die Wissenschaftsentwicklung in den Blick und werden zum Objekt der Forschung. Die kontextuelle Disziplingeschichtsschreibung hat demnach eher die Wissenschaftlergruppe als den Einzelwissenschaftler im Visier (Peckhaus und Thiel 1999, S. 13).

In der Folge wird die Disziplingeschichte in ein neues Licht gerückt. So hält etwa Hubert Laitko (1999) fest, dass nun nicht allein der ,patriarchalische Charakter der traditionellen Wissenschaft" (Laitko 1999, S. 24) deutlich werde; es zeige sich eben auch, dass die „Leistungen ganzer Kollektive schöpferischer Wissenschaftler [...] stillschweigend wenigen großen Gestalten zugeschrieben“ (Laitko 1999, S. 26) und die Rekonstruktion des disziplinären Wissens fast durchweg ,,vom Standpunkt der Sieger" (Laitko 1999, S. 26) aus betrieben werde. Mit problematischen Formen der Attribuierung sei freilich nicht allein bei der Reflexion innerdisziplinärer Entwicklungen zu rechnen; die systematische Berücksichtigung von Kontexten müsse 
künftig dazu führen, auch benachbarte Disziplinen stärker zu berücksichtigen und sie als konkurrierende Akteur*innen in den Blick zu nehmen (vgl. Laitko 1999).

Und tatsächlich - es tut sich etwas. Dies gilt sogar für die Philosophie, die den Kult der Meisterdenker besonders lange betrieb (vgl. Thomä et al. 2015). So ist Dieter Henrich bei der Erforschung des Frühidealismus in den 1980er-Jahren neue Wege gegangen und hat das komplexe Geflecht von Einzelpersonen, Problemstellungen und Kommunikationsbeziehungen untersucht (vgl. Henrich 1991). Dieser Zugang wurde von Henrich und seinen Mitstreiter*innen zu einem eigenständigen methodischen Zugang ausgearbeitet, der unter dem Namen „Konstellationsforschung“ firmiert (vgl. Mulsow und Stamm 2005). Einen anderen Zugang wählt Kurt Flasch (2008). Unter dem Titel „Kampfplätze der Philosophie“ (Flasch 2008) rekonstruiert er die Geschichte des abendländischen Denkens als Abfolge von Konflikten, die zwischen exponierten Vertretern der Philosophie ausgetragen werden. Auch wenn diese Form der Historiographie noch dem Narrativ der (männlichen) Meisterdenker verpflichtet bleibt, treten hier Dissens und Konflikt als Motor der Wahrheitssuche deutlich hervor; auch bei K. Flasch ist es nicht länger das isolierte Erkenntnissubjekt. An dessen Stelle treten Auseinandersetzungen, ,Denk-Situationen“ und ,Wahrheitskämpfe“ (Flasch 2008, S. 7).

Dass sich auch in der Soziologie verwandte Bestrebungen beobachten lassen, muss kaum überraschen. Von Überlegungen Georg Simmels und Karl Mannheim inspiriert, haben Georg Kneer und Stephan Moebius (2010) einen Band mit ausgewählten „Soziologische[n] Kontroversen“ (Kneer und Moebius 2010) zusammengestellt. Sie legen damit Beiträge zu einer ,,anderen Geschichte der Wissenschaft vom Sozialen“ vor, lenken den Blick auf die Vielzahl von Konflikten und plädieren dafür, mit einer Verschmutzung wissenschaftlicher Diskurse zu rechnen. Und sie warnen vor einer machtblinden Form der Disziplingeschichtsschreibung, einer Variante, ,,bei der die einseitige Parteinahme für die ,Sieger“ der Wissenschaftskontroversen erfolgt, ohne dass die Argumente der ,Verlierer' und deren zeitbedingter Ideen- und Problemhintergrund Berücksichtigung finden - und ohne angemessen zu reflektieren, dass die Zuweisung des Sieger- beziehungsweise Verliererstatus selbst wiederum perspektiv- und kontextabhängig ist“" (Kneer und Moebius 2010, S. 9).

\section{Zürich, 2000}

Das Problembewusstsein für die Unzulänglichkeiten konventioneller Varianten der Disziplingeschichtsschreibung wächst indes auch in der Erziehungswissenschaft. Schon Mitte der 1980er Jahre werden hier erste Beiträge vorgelegt, die dafür werben, eine reflexive Wissenschaftspraxis einzuüben. So spricht sich Ulrich Herrmann (1989) dafür aus, neue „Institutionen und Formen der Selbstreflexion“ zu entwickeln (Herrmann 1989, S. 2). Dass Fallstudien zur pädagogischen Wissenschaftsgeschichte genau dies zu leisten vermögen, demonstrieren Jürgen Oelkers (1989) und HeinzElmar Tenorth (1989). J. Oelkers (1989) rekonstruiert das „Ende des Herbartianismus" und erläutert, wie erbittert seinerzeit die Kämpfe um das Erbe Herbarts geführt wurden. Ähnlich abgeklärt erläutert H.-E. Tenorth (1989) am Beispiel der Rezeption 
der empirischen Erziehungswissenschaft um 1900, wie wissenschaftliche Konflikte bisweilen entschieden werden:

Die Gestalt der wissenschaftlichen Pädagogik [...] gehorcht nicht der Sachlogik des Erziehungsphänomens, sondern historischen Bedingungen, sie ist eine durch Selektionen, Negationen und institutionelle Ausgrenzung erzeugte, kontingente Gestalt der Wissenschaft, die [...] deshalb herrschend wird, [...] weil sie ein Umfeld findet, das den Opponenten institutionell und politisch abweist (Tenorth 1989, S. 337).

Obwohl in der Erziehungswissenschaft schon recht früh innovative Beiträge zu einer neuen Form der Disziplingeschichtsschreibung vorlagen, wurden diese doch nicht stilbildend. Erst mit einer gewissen Verzögerung kommt es um die Jahrtausendwende zu einem neuen Anlauf.

Vor zwanzig Jahren lud die Redaktion der Zeitschrift für pädagogische Historiographie dazu ein, die Konturen einer weniger unterkomplexen Form der Disziplingeschichte zu skizzieren. Zu diesem Zweck intensivierten die Autor*innen die Suche nach ,wissenschaftliche[n] Alternativen zur linearen hagiographischen Geschichtsschreibung" (Tröhler 2001, S. 26) und erinnerten nicht allein an Thomas Kuhns Studien; sie diskutierten überdies die Optionen des französischen Poststrukturalismus und problematisierten die Gegenüberstellung von Ideen- und Sozialgeschichte (vgl. Oelkers 2001; Winkler 2001). Dergestalt treten einige jener Anforderungen hervor, denen eine Disziplingeschichte der Erziehungswissenschaft neuer Prägung zu entsprechen hätte: Zunächst müsste sie sich von allen erzieherischen Ambitionen verabschieden und einer ,ent-pädagogisierte[n] Geschichtsschreibung“ (Tröhler 2001, S. 26) den Vorzug geben. Ferner sollte die Erforschung der Vergangenheit der Disziplin nicht mit dem Interesse an deren Stabilisierung betrieben werden; die Spurensuche sollte also keiner verdeckten Teleologie zuarbeiten (vgl. Tröhler 2001, S. 28). Vielmehr biete das historische Material der Disziplin die Möglichkeit der Befremdung des Eigenen und Vertrauten. Kaum weniger wichtig sei die konsequent betriebene Kontextualisierung: Sie lenke die Aufmerksamkeit nicht nur auf machtvolle Diskurse, hegemoniale Kämpfe und die Bedeutung von Kontroversen, sondern auch auf die sich fortwährend verändernden Bedingungen, unter denen die Wahrheitsspiele ausgetragen werden. Schließlich gelte es, die Frage zu diskutieren, „für wen die Geschichte [der eigenen Disziplin] erforscht und beschrieben"werden solle (Casale et al. 2004, S. 7).

Dabei zeichnete sich ab, wie anspruchsvoll das Unterfangen ist, nicht allein die Wissensbestände einer Disziplin systematisch zu erforschen, sondern auch die diskursiven Praktiken, über die dieses Wissen erzeugt wird. So schwierig es ist, die sich fortwährend verändernden Regeln des Archivs zu erschließen, über welche die Wissensbestände des pädagogischen Diskurses erzeugt werden, so kompliziert (und heikel) ist das Unternehmen, die sozialen Praktiken $\mathrm{zu}$ erschließen, die den $\mathrm{Zu}$ gang zum wissenschaftlichen Feld regeln. Wertet man etwa unter den Beiträgen zur Subjektivierungsforschung jene aus, welche die Formierung des Erkenntnissubjekts zum Gegenstand machen (vgl. Rieger-Ladich 2017), erscheinen diese nicht nur als eindrucksvolle Bestätigung für Michel Foucaults (1991) These der Kontrolle, Reglementierung und Kanalisierung der Produktion von Diskursen; sie zeigen auch, wie 
schwierig das Projekt ist, die sozialen Praktiken zu erforschen, über die der Zugang zur scientific community organisiert wird (vgl. Etzemüller 2019). P. Bourdieu (1988) Beobachtung, dass die Untersuchung des akademischen Unbewussten bei den betroffenen Akteur*innen kaum einmal auf große Gegenliebe stößt, hat auch mehr als 30 Jahre später nichts von ihrer Gültigkeit eingebüßt. Allen Bemühungen um wissenschaftliche Reflexivität zum Trotz, gibt es noch immer beträchtlichen Aufklärungsbedarf über die soziale Logik der Academia (vgl. Etzemüller 2019; Honegger 2007). Und dies eben auch deshalb, weil viele Praktiken - etwa der Ausschluss von Personen und die Marginalisierung von Themen - häufig informell betrieben werden.

\section{Tübingen, 2018}

Das Archiv gilt es somit nicht nur bezüglich seiner Struktur zu erforschen, es muss auch auf seine Lücken hin untersucht werden. Die Aufforderung Jacques Derridas (2009), in den Lücken des Archivs zu lesen und dessen Leerstellen zu erkunden, gilt auch für die Erziehungswissenschaft. Ähnlich wie dies von feministischen Wissenschaftskritiker*innen längst praktiziert wird, gilt es auch für die Vertreter*innen des pädagogischen Diskurses, diesen auf seine systematischen Auslassungen hin zu untersuchen - also Gesagtes wie auch Ungesagtes zum Gegenstand zu machen (vgl. Rohstock 2019; Brumlik 2019). Ferner ist es dringend geboten, nach empirischem Material zu fahnden, das es erlaubt, die etablierten Erzählmuster zu durchkreuzen und Gegengeschichten zu erzählen. Auf diese Weise lässt sich nicht nur das Gedächtnis für die hegemonialen Kämpfe um die Ordnung des pädagogischen Diskurses schärfen, auch die dominanten Narrative können dabei identifiziert und freigelegt werden. Den identitätsstiftenden Erzählungen über die Geschichte unserer Disziplin wäre daher mit einer Kultivierung jener „Hermeneutik des Verdachts“ (Ricoeur 2009) zu begegnen, die auch in den Nachbardisziplinen zu produktiven Verfremdungen geführt hat.

Nicht weniger wichtig als die Aufdeckung der hegemonialen Kämpfe, die um die Ordnung des pädagogischen Diskurses geführt werden, ist freilich der Versuch, diesen zu überschreiten - und neue Aussagesysteme zu etablieren sowie alternative Narrative zu erproben. Energien gilt es daher nicht allein auf die Kritik der herrschenden Ordnung disziplinären Wissens zu richten, sondern auch in die Erprobung alternativer Arrangements zu investieren. Auch im Nachdenken über die Geschichte der Erziehungswissenschaft sollten wir künftig den Konjunktiv-Plusquamperfekt kultivieren (vgl. Rieger-Ladich 2015) und Fragen der folgenden Art aufwerfen: Wie hätte sich der Gang der Geschichte entwickelt, wenn sich unsere Fachvertreter 1933 als weniger korrumpierbar erwiesen hätten? Welche Verläufe hätte die Theoriearbeit genommen, wenn es nach 1945 nicht zu einer Dominanz der geisteswissenschaftlichen Pädagogik gekommen wäre (vgl. Berg et al. 2004)? Welches Profil hätte die Erziehungswissenschaft ausgeprägt, wenn sich die Hochschulen im deutschsprachigen Raum als weniger patriarchal erwiesen hätten? Wie sähe das Curriculum heute aus, wenn die Verstrickung in den Kolonialismus früher (an-)erkannt und als Anlass zur Selbstkritik begriffen worden wäre? Auf welche Erkenntniswerkzeuge könnten 
wir zugreifen, wenn die Theorieofferten des Marxismus, der Psychoanalyse, der Ideologiekritik, des Feminismus, der Queer- und der Postcolonial Studies hierzulande früher ergriffen worden wären? Welche Debattenkultur würde unsere Disziplin heute charakterisieren, wenn jüngere Kolleg*innen, die eine akademische Karriere anstreben, sich weniger stark ausgeprägten Erwartungen von Loyalität gegenübersähen? Wenn hierzulande die Habilitation Originalität und Unkonventionalität belohnen würde statt habituelle Passung (vgl. Brenner 1993)? Wie sähe das intellektuelle Profil der Erziehungswissenschaft aus, wenn es gelungen wäre, der Außensteuerung der Universität stärker entgegenzutreten? Wenn die Orientierung an einer Kultur des Messens, Zählens und Evaluierens von Beginn an entschlossener bekämpft worden wären (vgl. Münch 2009; Radtke 2008)? Und wenn wir schon früher damit begonnen hätten, neue, kollaborative Formen der Arbeit zu erproben?

Solche Fragen auch nur aufzuwerfen, ist bereits geeignet, die herrschenden Verhältnisse als kontingent zu markieren und daran zu erinnern, dass auch die Ordnung des pädagogischen Diskurses veränderbar ist, dass sie kein getreues Abbild des pädagogischen Feldes darstellt, sondern sich hegemonialen Kämpfen verdankt. Überdies können solche Fragen zu Stimulanzien werden, um über alternative Praktiken des Archivierens nachzudenken. Das Ziel dieser kollektiven (Selbst-)Reflexion bestünde darin, ein Archiv disziplinären Wissens anzulegen, das den pädagogischen Diskurs fortwährend mit Komplexität anreichert. Bei der Organisation eines solchen Archivs erziehungswissenschaftlichen Wissens wäre zu berücksichtigen, dass sich heute nicht verlässlich sagen lässt, welche Erkenntniswerkzeuge und Theorietraditionen wir morgen und übermorgen benötigen. Als Maxime sollte daher gelten, ein möglichst vielstimmiges Archiv anzulegen, also eines, das von Pluralität und Diversität geprägt ist. Es gilt, möglichst unterschiedliche theoretische, methodische und empirische Zugänge zu kultivieren und „,vorzuhalten“ (vgl. Fohrmann 2013).

Auch innerhalb der Erziehungswissenschaft sollten wir damit rechnen, dass es immer wieder zu überraschenden ,Konstellationen“ (Benjamin 2010) zwischen vergangenen und künftigen Problemlagen kommt, dass aktuelle Krisenphänomene ältere Wissensbestände in einem veränderten Licht erscheinen lassen. Allen Versuchen der Verknappung, der Ausdünnung und der Einschränkung der Reflexion gilt es daher entgegenzutreten - mithin allen Bestrebungen, die intellektuellen Ressourcen zu begrenzen. In Unkenntnis dessen, was den pädagogischen Diskurs in der Zukunft einmal herausfordern wird, erscheint es mir dringend geboten, dafür Vorkehrungen zu treffen, dass die Reflexion pädagogischer Fragestellungen mit Komplexität angereichert und bei deren Bearbeitung eine Vielzahl unterschiedlicher Perspektiven eingenommen werden kann. „Häresie“ und „Devianz“ wären daher nicht länger als Makel zu bewerten, sondern sollten als akademische Tugenden, als unverzichtbare Elemente einer widerständigen Wissenschaftskultur begriffen werden, die den Dissens als erkenntnisstiftendes Moment erkennt.

Damit kehre ich ein letztes Mal zum Feld der Kunst zurück. Hier werden derzeit ähnliche Fragestellungen diskutiert. Zeitgenössische Kunsttheoretiker*innen wie Natalie Bayer, Oliver Marchart, Carmen Mörsch und Mark Terkessidis radikalisieren die Institutionskritik noch weiter und fragen danach, wie eine emanzipatorische Ausstellungspraxis aussehen könnte. Auch sie suchen den musealen Strategien der Selbstnaturalisierung entgegenzutreten und eine gegenhegemoniale Praxis zu entwi- 
ckeln. So sprechen sich Natalie Bayer und Mark Terkessidis (2017) für ,,multiperspektivische Erzählungen, enthierarchisierende Geschichtsbilder und selbstbestimmte[.] Geschichts-/Darstellungen" aus (Bayer und Terkessidis 2017, S. 55). Sie zeigen dabei das Museum als eine autoritäre Institution und versuchen, dessen Selbstauratisierung zu durchkreuzen. Und sie tun dies im Wissen darum, dass auch die Kritik der Kritik nie davor gefeit ist, zu einer schalen Überbietungsgeste zu werden. Auch das lässt sich von den Performances Andrea Frasers lernen. Sie treibt die Reflexivität immer weiter voran - und steht damit in der Gefahr, den Ort zu unterminieren, von dem aus sie spricht (vgl. Fraser 2005b) Vor vergleichbaren Herausforderungen stehen wir auch innerhalb des wissenschaftlichen Feldes: auch hier kann das engagiert vorgetragene Plädoyer für Reflexivität zur Spielmarke werden (vgl. Lynch 2012). Es gilt daher neue Formen der Selbstbeobachtungen zu erproben, die ohne (vermeintliche) Gewissheiten auskommen und die auch die Geste der Kritik auf den Prüfstand stellen - eben: kritisch hinterfragen (vgl. Rieger-Ladich 2020; Wortmann 2019).

Funding Open Access funding provided by Projekt DEAL.

Open Access Dieser Artikel wird unter der Creative Commons Namensnennung 4.0 International Lizenz veröffentlicht, welche die Nutzung, Vervielfältigung, Bearbeitung, Verbreitung und Wiedergabe in jeglichem Medium und Format erlaubt, sofern Sie den/die ursprünglichen Autor(en) und die Quelle ordnungsgemäß nennen, einen Link zur Creative Commons Lizenz beifügen und angeben, ob Änderungen vorgenommen wurden.

Die in diesem Artikel enthaltenen Bilder und sonstiges Drittmaterial unterliegen ebenfalls der genannten Creative Commons Lizenz, sofern sich aus der Abbildungslegende nichts anderes ergibt. Sofern das betreffende Material nicht unter der genannten Creative Commons Lizenz steht und die betreffende Handlung nicht nach gesetzlichen Vorschriften erlaubt ist, ist für die oben aufgeführten Weiterverwendungen des Materials die Einwilligung des jeweiligen Rechteinhabers einzuholen.

Weitere Details zur Lizenz entnehmen Sie bitte der Lizenzinformation auf http://creativecommons.org/ licenses/by/4.0/deed.de.

\section{Literatur}

\section{Verwendete Literatur}

Baecker, D. (2017). Von der Kunst, die Wirklichkeit unmöglich zu machen. Zu Heiner Müller. Merkur. Deutsche Zeitschrift für europäisches Denken, 813, 16-28.

Barthes, R. (2012). Mythen des Alltags. Berlin: Suhrkamp. vollständige Ausgabe

Bayer, N., \& Terkessidis, M. (2017). Über das Reparieren hinaus. Eine antirassistische Praxeologie des Kuratierens. In N. Bayer, B. Kazeem-Kaminski \& N. Sternfeld (Hrsg.), Kuratieren als antirassistische Praxis (S. 50-71). Berlin und Boston: Walter de Gruyter.

Benjamin, W. (2010). Über den Begriff der Geschichte. Werke und Nachlass. Kritische Gesamtausgabe, Bd. 19. Berlin: Suhrkamp. Herausgegeben von Gérard Raulet

Bennett, T. (2008). Der bürgerliche Blick. Das Museum und die Organisation des Sehens. In D. von Hantelmann \& C. Meister (Hrsg.), Die Ausstellung. Politik eines Rituals. Berlin-Zürich: diaphanes.

van den Berg, K. (2015). Kritik, Protest, Poiesis. Künstler mischen sich ein - von 1970 bis heute. In A. Nassehi \& P. Felixberger (Hrsg.), Kursbuch 182. Das Kursbuch. Wozu? 50 Jahre Jubiläumsedition (S. 171-188). Hamburg: Sven Murmann. 
Berg, C., Herrlitz, H.-G., \& Horn, K.-P. (Hrsg.). (2004). Kleine Geschichte der Deutschen Gesellschaft für Erziehungswissenschaft. Eine Fachgesellschaft zwischen Wissenschaft und Politik. Wiesbaden: Springer VS.

Boltanski, L., \& Chiapello, È. (2003). Der neue Geist des Kapitalismus. Konstanz: UVK.

Bourdieu, P. (1987). Die feinen Unterschiede. Kritik der gesellschaftlichen Urteilskraft. Frankfurt am Main: Suhrkamp.

Bourdieu, P. (1988). Homo academicus. Frankfurt am Main: Suhrkamp.

Bourdieu, P. (1995). Sozialer Raum und „Klassen“. Leçon sur la leçon. Zwei Vorlesungen. Frankfurt am Main: Suhrkamp.

Bourdieu, P. (1999). Regeln der Kunst. Genese und Struktur des literarischen Feldes. Frankfurt am Main: Suhrkamp.

Bourdieu, P., \& Haacke, H. (1995). Freier Austausch. Für die Unabhängigkeit der Phantasie und des Denkens. Frankfurt am Main: Fischer.

Brenner, P.J. (1993). Habilitation als Sozialisation. In P. J. Brenner (Hrsg.), Geist, Geld, Wissenschaft. Arbeits- und Darstellungsformen von Literaturwissenschaft (S. 318-356). Frankfurt am Main: Suhrkamp.

Brüggmann, F. (2020). Institutionskritik im Feld der Kunst. Entwicklung - Wirkung - Veränderungen. Bielefeld: transcript.

Brumlik, M. (2019). Vergangenheit, die nicht vergehen will. Wie sich die deutsche Erziehungswissenschaft mit nationalsozialistischen Altvorderen plagt. In M. Rieger-Ladich, A. Rohstock \& K. S. Amos (Hrsg.), Erinnern, umschreiben, vergessen. Die Stiftung des disziplinären Gedächtnisses als soziale Praxis (S. 277-289). Weilerswist: Velbrück Wissenschaft.

Casale, R., Tröhler, D., \& Oelkers, J. (2004). Lebenslanges Lernen in historischer Perspektive. Drei Beispiele für ein altes Konzept. Zeitschrift für Pädagogik, 50(1), 21-37.

Dath, D. (2018). Karl Marx. Stuttgart: Reclam. 100 Seiten

Derrida, J. (2009). Dem Archiv verschrieben. In K. Ebeling \& S. Altekamp (Hrsg.), Archivologie. Theorien des Archivs in Philosophie, Medien und Künsten (S. 29-60). Berlin: Kadmos.

Draxler, H. (2007). Gefährliche Substanzen. Zum Verhältnis von Kritik und Kunst. Berlin: b-books.

Etzemüller, T. (Hrsg.). (2019). Der Auftritt. Performanz in der Wissenschaft. Bielefeld: transcript.

Flasch, K. (2008). Kampfplätze der Philosophie. Große Kontroversen von Augustin bis Voltaire. Frankfurt am Main: Vittorio Klostermann.

Fleck, L. (1980). Entstehung und Entwicklung einer wissenschaftlichen Tatsache. Einführung in die Lehre vom Denkstil und Denkkollektiv. Frankfurt am Main: Suhrkamp.

Fohrmann, J. (2013). Weltgesellschaft und Nationalliteratur (am Beispiel der Germanistik). Merkur. Deutsche Zeitschrift für europäisches Denken, 770, 607-618.

Foucault, M. (1991). Die Ordnung des Diskurses. Mit einem Essay von Ralf Konersmann. Frankfurt am Main: Fischer.

Fraser, A. (2005a). From the critique of institutions to an institute of critique. Artforum International, 44, $278-283$.

Fraser, A. (2005b). Jenseits der Institutionskritik. Ein Vortrag im Los Angeles County Museum of Art. Texte zur Kunst, 59, 41-53.

Fraser, A. (2008). Es geht um Kultur. In B. Bismarck, T. Kaufmann \& U. Wuggenig (Hrsg.), Nach Bourdieu: Visualität, Kunst und Politik (S. 289-302). Wien: Turia \& Kant.

Gabriel, G. (2019). Präzision und Prägnanz. Logische, rhetorische, ästhetische und literarische Erkenntnisformen. Paderborn: mentis.

von Hantelmann, D., \& Meister, C. (Hrsg.). (2010). Die Ausstellung. Politik eines Rituals. Zürich/Berlin: diaphanes.

Haraway, D. (1995). Die Neuerfindung der Natur. Primaten, Cyborgs und Frauen. Frankfurt am Main: Campus.

Henrich, D. (1991). Konstellationen. Konstellationen. Probleme und Debatten am Ursprung der idealistischen Philosophie (1789-1795). Stuttgart: Klett-Cotta.

Herrmann, U. (1989). Die ,Kommission Wissenschaftsforschung ' der Deutschen Gesellschaft für Erziehungswissenschaft. In P. Zedler \& E. König (Hrsg.), Rekonstruktionen pädagogischer Wissenschaftsgeschichte. Fallstudien, Ansätze, Perspektiven (S. 1-19). Weinheim: Deutscher Studien Verlag.

Honegger, C. (2007). Konkurrenzverhältnisse. Disziplinen, wissenschaftliche Felder, epistemische Kulturen. In C. Honegger, H.-U. Jost, S. Burren \& P. Jurt (Hrsg.), Konkurrierende Deutungen des Sozialen. Geschichts-, Sozial- und Wirtschaftswissenschaften im Spannungsfeld von Politik und Wissenschaft (S. 19-41). Zürich: Chronos. 
Kneer, G., \& Moebius, S. (Hrsg.). (2010). Soziologische Kontroversen. Beiträge zu einer anderen Geschichte der Wissenschaft vom Sozialen. Berlin:: Suhrkamp.

Laitko, H. (1999). Disziplingeschichte und Disziplinverständnis. In V. Peckhaus \& C. Thiel (Hrsg.), Disziplinen im Kontext. Perspektiven der Disziplingeschichtsschreibung (S. 21-60). München: Fink.

Lepenies, W. (1978). Wissenschaftsgeschichte und Disziplingeschichte. Geschichte und Gesellschaft, 4, 437-451.

Lynch, M. (2012). Gegen Reflexivität als akademischer Tugend und Quelle privilegierten Wissens. Zeitschrift für qualitative Bildungs-, Beratungs- und Sozialforschung, 5(2), 273-309.

Marchart, O. (2005). Die Institution spricht. Kunstvermittlung als Herrschafts- und Emanzipationstechnologie. In B. Jaschke, C. Martinz-Turek \& N. Sternfeld (Hrsg.), Wer spricht? Autorität und Autorschaft in Austellungen (Bd. 1, S. 34-58). Wien: Turia + Kant.

Mulsow, M., \& Stamm, M. (Hrsg.). (2005). Konstellationsforschung. Frankfurt am Main: Suhrkamp.

Münch, R. (2009). Stratifikation der Hochschullandschaft. Zwischen Leistungswettbewerb und Machtlogik. Zeitschrift für Pädagogik, 51(2), 258-273.

Oelkers, J. (1989). Das Ende des Herbartianismus. Überlegungen zu einem Fallbeispiel der pädagogischen Wissenschaftsgeschichte. In P. Zedler \& E. König (Hrsg.), Rekonstruktionen pädagogischer Wissenschaftsgeschichte. Fallstudien, Ansätze, Perspektiven (S. 77-116). Weinheim: Deutscher Studien Verlag.

Oelkers, J. (2001). Ein Essay über den schwindenden Sinn des Gegensatzes von ,Ideengeschichte“ und ,Sozialgeschichte' in der pädagogischen Geschichtsschreibung. Zeitschrift für pädagogische Historiographie, 7(1), 21-25.

Peckhaus, V., \& Thiel, C. (1999). Kontextuelle Disziplingeschichtsschreibung. In V. Peckhaus \& C. Thiel (Hrsg.), Disziplinen im Kontext. Perspektiven der Disziplingeschichtsschreibung (S. 7-20). München: Fink.

Radtke, F.-O. (2008). Die außengeleitete Universität. WestEnd, 5(1), 117-133.

Raunig, G. (2007). Instituierende Praxen. Fliehen, Instituieren, Transformieren. Psychologie \& Gesellschaftskritik, 31(1), 81-92.

Reitz, T. (2016). Von der Kritik zur Konkurrenz. Die Umstrukturierung wissenschaftlicher Konflikte und ihre Wissenseffekte. sub urban. zeitschrift für kritische stadtforschung, 4(2/3), 37-58.

Ricoeur, P. (2009). Der Konflikt der Interpretationen: Ausgewählte Aufsätze (1960-1969). Freiburg im Breisgau: Alber.

Rieger-Ladich, M. (2009). Verstrickung und Verantwortung. Typen und Quellen wissenschaftlicher Reflexivität. In N. Ricken, H. Röhr, J. Ruhloff \& K. Schaller (Hrsg.), Umlernen. Festschrift für Käte Meyer-Drawe (S. 315-325). München: Fink.

Rieger-Ladich, M. (2010). Gedächtnis der Kämpfe. Neue Impulse für die Disziplingeschichtsschreibung. In C. Dietrich \& H.-R. Müller (Hrsg.), Die Aufgabe der Erinnerung in der Pädagogik (S. 227-243). Bad Heilbrunn: Klinkhardt.

Rieger-Ladich, M. (2014). Erkenntnisquellen eigener Art? Literarische Texte als Stimulanzien erziehungswissenschaftlicher Reflexion. Zeitschrift für Pädagogik, 3, 350-367.

Rieger-Ladich, M. (2015). Konjunktiv Plusquamperfekt. Alexander Kluge und Nicholson Baker. In A. Dörpinghaus \& B. Platzer (Hrsg.), Bildung an ihren Grenzen. Zwischen Bildungstheorie und Bildungsforschung (S. 189-204). Darmstadt: Wissenschaftliche Buchgesellschaft.

Rieger-Ladich, M. (2017). Situierte Subjekte. Über Wissenschaft als soziale Praxis. In O. Dörner, C. Iller, H. Pätzold, J. Franz \& B. Schmidt-Hertha (Hrsg.), Generation - Biografie - Lebenslauf (S. 21-35). Opladen: Barbara Budrich.

Rieger-Ladich, M. (2019). Bildungstheorien zur Einführung. Hamburg: Junius.

Rieger-Ladich, M. (2020). Abstieg vom Feldherrenhügel. Zum Ort kritischer Theoriebildung. In M. Harant \& P. Thomas (Hrsg.), Theorien! Horizonte für die Lehrerinnen- und Lehrerbildung (S. 17-27). Tübingen: Tübingen University Press.

Rieger-Ladich, M., Rohstock, A., \& Amos, K. (Hrsg.). (2019). Erinnern, Umschreiben, Vergessen. Die Stiftung des disziplinären Gedächtnisses als soziale Praxis. Weilerswist: Velbrück.

Rohstock, A. (2019). Vom NS-Statistiker zum bundesrepublikanischen Bildungsforscher. Friedrich Edding und seine Verstrickung in den Nationalsozialismus. In M. Rieger-Ladich, A. Rohstock \& K. Amos (Hrsg.), Erinnern, Umschreiben, Vergessen. Die Stiftung des disziplinären Gedächtnisses als soziale Praxis (S. 120-157). Weilerswist: Velbrück.

Rustemeyer, D. (1992). Identität als faktische Fiktion? Erziehungswissenschaft zwischen Modernisierung und Modernitätskrise. Beiheft der Zeitschrift für Pädagogik, 29, 187-188.

Rustemeyer, D. (2017). Ordnungen des Wirklichen. Weisen des Unterscheidens in Philosophie, Künsten und Wissenschaft. Freiburg und München: Karl Alber. 
Sonderegger, R. (2019). Vom Leben der Kritik. Kritische Praktiken und die Notwendigkeit ihrer geopolitischen Situierung. Wien: Zaglossus.

Steyerl, H. (2006). Die Institution der Kritik. Psychologie und Gesellschaftskritik, 31(1), 71-80.

Tenorth, H.-E. (1989). Versäumte Chancen. Zur Rezeption und Gestalt der empirischen Erziehungswissenschaft der Jahrhundertwende. In P. Zedler \& E. König (Hrsg.), Rekonstruktionen pädagogischer Wissenschaftsgeschichte. Fallstudien, Ansätze, Perspektiven (S. 317-343). Weinheim: Deutscher Studien Verlag.

Thiel, C. (1993). Neuere Überlegungen zur Geschichtsschreibung einzelwissenschaftlicher Disziplinen. In P. Janich (Hrsg.), Neue Entwicklungen in der methodischen Philosophie (S. 125-147). Frankfurt am Main: Suhrkamp.

Thomä, D., Schmid, U., \& Kaufmann, V. (2015). Der Einfall des Lebens: Theorie als geheime Autobiographie. München: Hanser.

Tröhler, D. (2001). Pädagogische Historiographie und Kontext. Zeitschrift für pädagogische Historiographie, 7(1), 26-34.

Winkler, M. (2001). Klassiker der Pädagogik. Überlegungen eines möglicherweise naiven Beobachters. Zeitschrift für pädagogische Historiographie, 7(2), 76-85.

Wortmann, K. (2019). Post-critical pedagogy as poetic practice: combining affirmative and critical vocabularies. Ethics and education, 14(4), 467-481.

\section{Weiterführende Literatur}

Arnold, M. (2004). Disziplin \& Initiation. Die kulturellen Praktiken der Wissenschaft. In M. Arnold \& R. Fischer (Hrsg.), Disziplinierungen. Kulturen der Wissenschaften im Vergleich (S. 18-52). Wien: Turia + Kant.

Bourdieu, P. (2002). Ein soziologischer Selbstversuch. Frankfurt am Main: Suhrkamp.

Fraser, A. (2011). Über die soziale Welt sprechen. Texte zur Kunst, 21(81), 88-93.

O’Doherty, B. (1996). In der weiße Zelle/Inside the White Cube. Berlin: Merve. Herausgegeben von W. Kemp. Mit einem Nachwort von M. Brüderlin 